\title{
The Atmospheric Scanning Electron Microscope (ASEM) Observes Axonal Segmentation and Synaptic Induction in Solution.
}

Chikara Sato ${ }^{1}$, Takaaki Kinoshita ${ }^{2}$, Takeshi Uemura $^{3}$, Kazumi Hiarno ${ }^{2}$, Masaaki Kawata ${ }^{1}$, Hidetoshi Nishiyama ${ }^{4}$, Mari Sato ${ }^{1}$, Tatsuhiko Ebihara ${ }^{1}$, Mitsuo Suga ${ }^{4}$, and Shoko Nishihara ${ }^{2}$

1. National Institute of Industrial Science and Technology (AIST), Tsukuba, 305-8566, Japan.

2. Faculty of Engineering, Soka University, 1-236 Tangi-machi, Hachioji, Tokyo 192-8577, Japan.

3. School of Medicine, Shinshu University 3-1-1 Asahi, Matsumoto, Nagano 390-8621, Japan.

4. JEOL Ltd., 1-2 Musashino 3-chome, Akishima, Tokyo 196-8558, Japan.

The new Atmospheric Scanning Electron Microscope (ASEM) is a Correlative Light-Electron Microscope (CLEM) [1]. Cell cultures of a few milliliters can be grown and differentiated directly in the removable ASEM dish, in a $\mathrm{CO}_{2}$ atmosphere if required. After fixation, the cells are imaged in situ, immersed in liquid and at atmospheric pressure, by optical microscopy (OM) and SEM in a fully correlative manner. Various dish coatings have been developed to increase the range of culturable cell types, which include primary neurons [2] allowing axonal segmentation and synapse formation to be investigated.

Axonal partitioning of neurons was correlated with specific cytoskeletal structures including microtubules. For this, isolated Drosophila primary neurons were grown on a poly-DL-ornithine-coated ASEM dish and immunolabeled for neuron markers HRP and BP102. Fluorescence microscopy demonstrated the localization of HRP in the whole axial fiber (Figure 1, A) and the specific localization of BP102 in the proximal region (B). ASEM revealed a hexagonal frame-like structure of BP102 at the boundary of the intra-axonal segment (C, arrow). Seven of the eleven axons examined displayed this structure, which has not been observed by OM. Using the location of BP102 to assign the intra-axonal boundary $(\mathrm{D}, \mathrm{F})$, allowed the microtubules in this region to be analyzed $(\mathrm{E}, \mathrm{G})$. In Figure 1E two tubulin bundles running alongside one another make contact at the intra-axonal boundary (arrow) and possibly elsewhere. Eight of the ten axons examined showed such contacts. In the other two axons, the immunolabeling was disconnected at the intra-axonal boundary ( $\mathrm{G}$, arrow) (Microsc. Microanal. in press). This may mean that the two tubulin bundles are separated, although the possibility that labeling is prevented by proteins bound to the $\alpha$-tubulins in this region cannot be excluded.

Synapse formation is critical for brain development and functions. Glutamate receptor delta 2 (GluRdelta2), which is predominantly expressed in cerebellar Purkinje cells, is essential for synapse formation in vivo. The extracellular N-terminal domain (NTD) of GluRdelta2 is known to interact with presynaptic neurexins through cerebellin 1 precursor protein and mediate synapse formation. However, the precise mechanisms involved are not fully understood, since synaptic structures are too small to be observed by OM. Mouse cerebellar neurons were cultured on a poly-L-lysine- and laminin- coated ASEM dish. On DIV6, both Sindbis-EGFP virus to visualize neurons and fluorescent magnetic beads coated with GluRdelta2-NTD-Fc were added to the cultures and incubated. After fixation, cells were immunostained against the presynaptic protein markers VGluT1 and/or Bassoon (Figure 2). Fluorescence microscopy showed presynaptic fibers leading to the magnetic beads and presynaptic Bassoon accumulated on the beads at the site of the contact. Subsequent ASEM suggested that presynaptic proteins, presumably of an indistinguishable neurite branch, surrounded each bead when a neurite was attached to it (Ultramicroscopy in press, http://dx.doi.org/10.1016/j.ultramic.2013.10.010). 
Controlling time and space for synaptogenesis by the use of magnetic beads, is expected to allow the molecular mechanism of synapse formation to be analyzed in the ASEM in the near future.

\section{References}

[1] H. Nishiyama et al, J Struct Biol 169 (2010), p. 438-449.

[2] Y. Maruyama et al, J Struct Biol 180 (2012), p. 259-270.
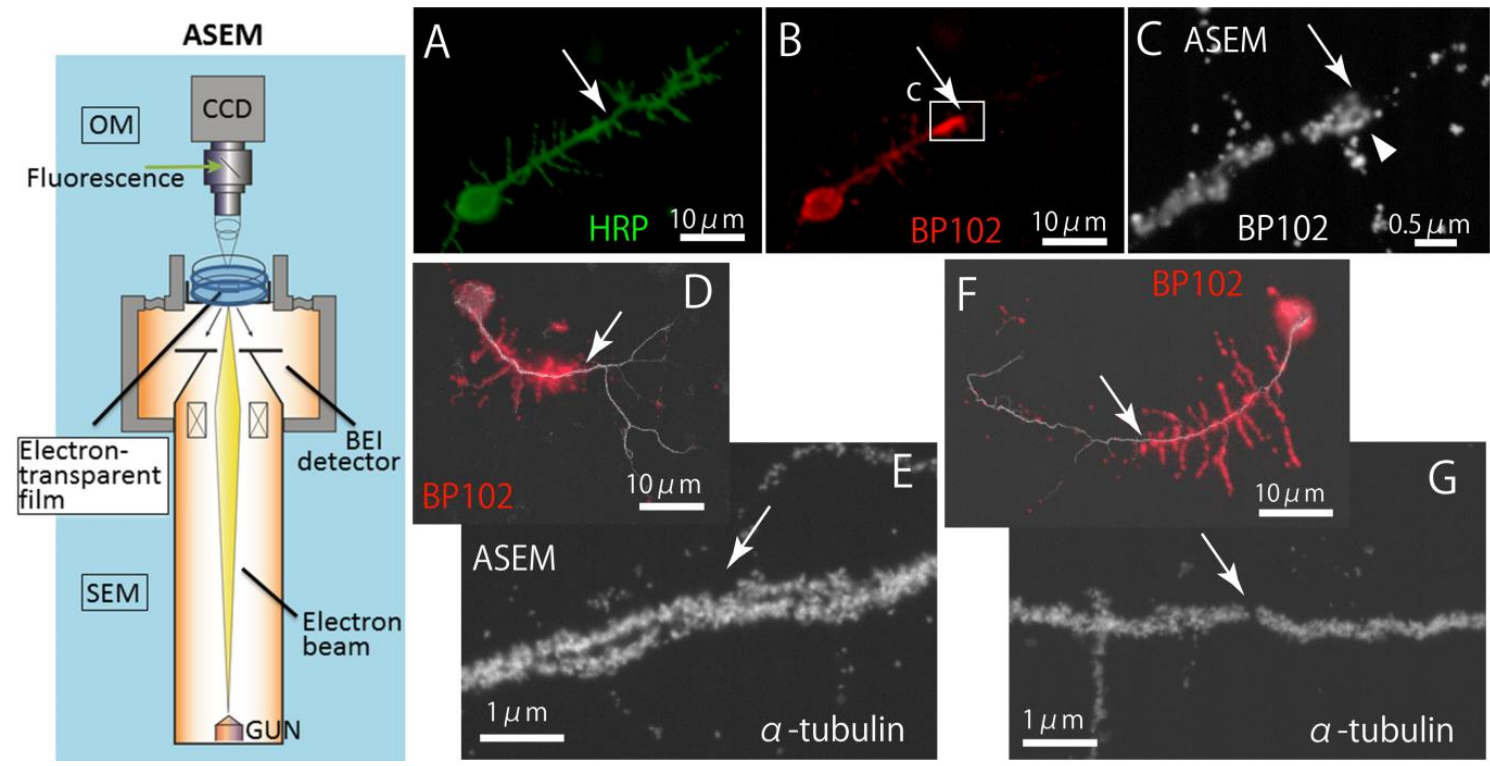

Figure 1. Axonal segmentation. (A) Localization of HRP epitopes (green). (B) Localization of BP102 epitopes (red). (C-G) ASEM. (C) ASEM of BP102. BP102 accumulates forming a special hexagonal frame-like structure at the intra-axonal boundary. (D-G) ASEM of $\alpha$-tubulin. In some cases, immunolabeled microtubule bundles make contact at the intra-axonal boundary $(\mathrm{E})$, in others they appear to be disconnected (G) (arrows) (Microsc. Microanal. in press).
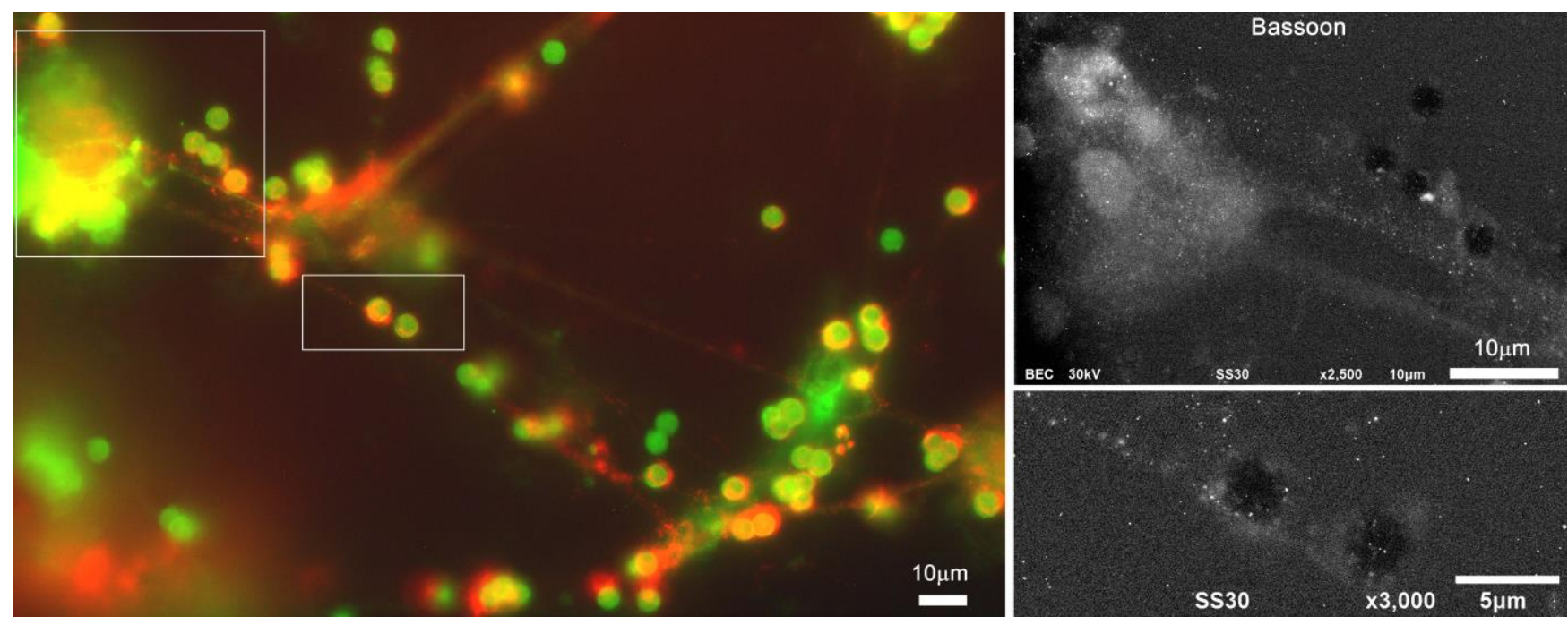

Figure 2. Distribution of Bassoon and VGluT1 (red) in relation to GluRdelta2-NTD-Fc-coated magnetic beads in mouse primary cerebellar cultures. The cytoplasm of EGFP-expressing neurons transfected by Sindbis-EGFP virus (green) and the magnetic beads (bright round features) are both imaged clearly. (Right) ASEM of Bassoon (grey-scale image); magnetic beads appear dark, while Bassoon is observed as a gathering of white dots presumably where axons connect to the beads (Ultramicroscopy in press). 